\title{
Serum and Vitreous Concentrations of Omentin-1 in Diabetic Retinopathy
}

\author{
Wencui Wan, Qiuming Li, Fengyan Zhang, Guangying Zheng, \\ Yong Lv, Guangming Wan, and Xuemin Jin \\ Department of Ophthalmology, The First Affiliated Hospital of Zhengzhou University, Zhengzhou, China \\ Correspondence should be addressed to Xuemin Jin; zzhjxm@126.com
}

Received 9 May 2015; Revised 8 June 2015; Accepted 11 June 2015

Academic Editor: Claudio Letizia

Copyright (c) 2015 Wencui Wan et al. This is an open access article distributed under the Creative Commons Attribution License, which permits unrestricted use, distribution, and reproduction in any medium, provided the original work is properly cited.

\begin{abstract}
Objective. Omentin, a new discovered adipokine, is implicated to inhibit inflammation. Inflammation is one important mechanism of diabetic retinopathy (DR). The purpose of this work was to evaluate serum and vitreous concentrations of omentin-1 in patients with diabetic retinopathy (DR). Methods. This study enrolled 204 diabetic patients (60 without DR, 49 with NPDR, and 95 with PDR) and 65 control subjects. Results. Serum and vitreous omentin-1 levels in PDR patients were markedly decreased compared with those in the other three groups. NPDR patients showed reduced vitreous omentin-1 compared with patients without DR. In addition, control subjects had significantly higher levels of serum and vitreous omentin-1 compared with diabetic patients without DR, NPDR patients, and PDR patients. In addition, serum/vitreous omentin-1 ratio was positively correlated with the development and severity of DR. Conclusion. Serum and vitreous omentin-1 levels, as well as serum/vitreous omentin-1 ratio, are correlated with the presence and severity of DR.
\end{abstract}

\section{Introduction}

Diabetic retinopathy (DR), the most serious diabetic complication, is estimated to account for $4.8 \%$ of global blindness [1]. Although major research has been concentrated on the elucidation of its pathogenesis, the clear mechanism of DR is still unknown. Within the factors involved in the mechanism of DR, inflammation is paid more attention during the past decade [2]. A multitude of inflammatory mediators have been demonstrated to be involved in the mechanism of diabetic retinopathy [3].

Omentin-1, a novel visceral fat depot-specific secretary protein, is produced/synthesized by the visceral stromal vascular cells [4]. Omentin-1 could increase insulinstimulated glucose uptake and Akt phosphorylation in human adipocytes [4]. Decreased omentin-1 concentrations were found in patients with insulin resistance (IR) [5] and diabetes mellitus [6]. Recently, omentin-1 was demonstrated to play an anti-inflammatory role in vascular smooth cells [7]. Inflammation is correlated with the development of DR.
Therefore, omentin-1 is hypothesized to play a role in DR development.

To our knowledge, the role of omentin-1 in the pathogenesis of DR has not been examined previously. With this background in mind, we designed this study to investigate the association of serum and vitreous omentin-1 levels with the presence of DR.

\section{Materials and Methods}

2.1. Patients. A consecutive population of 204 patients who were diagnosed with type 2 diabetes mellitus (T2DM) and underwent vitrectomy were divided into T2DM patients without DR group $(n=60)$, non-PDR (NPDR) group $(n=49)$, and PDR group $(n=95)$. Subjects were excluded if they had chronic systemic disease, cancers, ocular disorders, previous intraocular surgery or laser treatment, photocoagulation, and intravitreal hemorrhages obvious vitreal hemorrhage within the previous 3 months. Sixty-five patients who underwent vitrectomy for retinal detachment 
TABLE 1: Various characteristics of diabetic patients and controls.

\begin{tabular}{|c|c|c|c|c|c|}
\hline & \multirow{2}{*}{ Controls } & \multicolumn{3}{|c|}{ Diabetic patients } & \multirow{2}{*}{$P$} \\
\hline & & Without DR & NPDR & PDR & \\
\hline$n$ & 65 & 60 & 49 & 95 & \\
\hline Age (years) & $57.85 \pm 6.90$ & $58.67 \pm 9.00$ & $58.16 \pm 12.66$ & $57.32 \pm 10.96$ & 0.872 \\
\hline Gender (M/F) & $32 / 33$ & $25 / 35$ & $23 / 26$ & $45 / 50$ & 0.852 \\
\hline $\mathrm{BMI}\left(\mathrm{Kg} / \mathrm{m}^{2}\right)$ & $23.77 \pm 1.59$ & $23.83 \pm 3.00$ & $24.32 \pm 3.94$ & $24.41 \pm 3.22$ & 0.468 \\
\hline SBP (mmHg) & $124.23 \pm 11.45$ & $141.75 \pm 21.74^{\mathrm{a}}$ & $140.51 \pm 21.44^{\mathrm{a}}$ & $140.05 \pm 23.44^{\mathrm{a}}$ & $<0.001$ \\
\hline DBP (mmHg) & $83.11 \pm 7.63$ & $89.25 \pm 9.69^{\mathrm{a}}$ & $87.37 \pm 13.48^{\mathrm{a}}$ & $87.11 \pm 12.20^{\mathrm{a}}$ & 0.016 \\
\hline HbAlc (\%) & $5.03 \pm 0.71$ & $7.76 \pm 0.95^{\mathrm{a}}$ & $7.82 \pm 1.03^{\mathrm{a}}$ & $7.82 \pm 1.30^{\mathrm{a}}$ & $<0.001$ \\
\hline FPG (mmol/L) & $5.11 \pm 0.40$ & $8.29 \pm 1.86^{\mathrm{a}}$ & $8.59 \pm 1.50^{\mathrm{a}}$ & $8.10 \pm 1.66^{\mathrm{a}}$ & $<0.001$ \\
\hline $\mathrm{TC}(\mathrm{mmol} / \mathrm{L})$ & $5.19 \pm 0.89$ & $5.38 \pm 1.40$ & $5.33 \pm 1.10$ & $5.33 \pm 1.28$ & 0.822 \\
\hline $\mathrm{TG}(\mathrm{mmol} / \mathrm{L})$ & $1.17 \pm 0.31$ & $1.81 \pm 0.58^{\mathrm{a}}$ & $1.85 \pm 0.55^{\mathrm{a}}$ & $2.03 \pm 0.62^{\mathrm{a}}$ & 0.002 \\
\hline LDL-C (mmol/L) & $3.35 \pm 0.73$ & $3.39 \pm 1.06$ & $3.36 \pm 0.92$ & $3.52 \pm 1.05$ & 0.643 \\
\hline HDL-C (mmol/L) & $1.45 \pm 0.27$ & $1.42 \pm 0.40$ & $1.43 \pm 0.33$ & $1.26 \pm 0.30^{\mathrm{abc}}$ & 0.001 \\
\hline
\end{tabular}

${ }^{\mathrm{a}} P<0.05$ versus control; ${ }^{\mathrm{b}} P<0.05$ versus diabetic patients without $\mathrm{DR} ;{ }^{\mathrm{c}} \mathrm{P}<0.05$ versus NPDR patients.

TABLE 2: Serum and vitreous omentin-1 levels in controls, diabetic patients without DR, NPDR patients, and PDR patients.

\begin{tabular}{|c|c|c|c|c|c|}
\hline $\begin{array}{l}\text { Omentin-1 } \\
(\mathrm{ng} / \mathrm{mL})\end{array}$ & $\begin{array}{l}\text { Controls } \\
(n=65) \\
\end{array}$ & $\begin{array}{l}\text { Without DR } \\
\quad(n=60)\end{array}$ & $\begin{array}{c}\text { NPDR } \\
(n=49) \\
\end{array}$ & $\begin{array}{c}\text { PDR } \\
(n=95) \\
\end{array}$ & $P$ value \\
\hline Serum & $\begin{array}{c}208.31 \\
(164.20-251.20)^{\mathrm{bc}}\end{array}$ & $\begin{array}{c}184.41 \\
(142.50-206.14)^{\mathrm{a}}\end{array}$ & $\begin{array}{c}166.97 \\
(132.96-199.21)^{\mathrm{a}}\end{array}$ & $\begin{array}{c}139.96 \\
(119.28-157.87)^{\mathrm{abc}}\end{array}$ & $<0.001$ \\
\hline Vitreous & $96.00(75.24-112.64)^{\mathrm{bc}}$ & $81.46(67.84-96.39)^{\mathrm{ac}}$ & $64.28(53.08-74.45)^{\mathrm{ab}}$ & $50.36(39.91-57.73)^{\mathrm{abc}}$ & $<0.001$ \\
\hline Serum/vitreous ratio & $1.99(1.72-2.63)^{\mathrm{c}}$ & $2.17(1.82-2.57)^{\mathrm{c}}$ & $2.72(2.08-3.37)^{\mathrm{ab}}$ & $2.88(2.31-3.48)^{\mathrm{abc}}$ & $<0.001$ \\
\hline
\end{tabular}

${ }^{\mathrm{a}} P<0.05$ versus control; ${ }^{\mathrm{b}} P<0.05$ versus diabetic patients without $\mathrm{DR} ;{ }^{\mathrm{c}} P<0.05$ versus NPDR patients.

with no systemic disease were recruited in the control group. The control group matched with T2DM patients in age, gender, and BMI.

The study was planned according to the ethics guidelines of the Helsinki Declaration and was approved by the Institutional Research Ethics Board of our university. All patients gave written informed consent regarding participation in this study.

2.2. Measurements. Serum was obtained from blood samples by centrifugation and was stored at $-80^{\circ} \mathrm{C}$ until analysis. Vitrectomy samples (approximately $0.3 \mathrm{~mL}$ ) were got using a standardized vitrectomy technique with manual suction before opening the infusion set. The serum and vitreous samples were analyzed for omentin-1 using commercially available enzyme-linked immunosorbent assay (Cusabio Biotech Corporation, USA).

2.3. Statistical Analysis. Data are presented as means \pm SD or median (interquartile range). Kolmogorov-Smirnov test was utilized to determine data normality. The differences of characteristics between patients with PDR and NPDR, diabetic patients without DR, and control subjects were compared using Chi-square tests, one-way ANOVA, or Kruskal-Wallis test. Statistical analysis was carried out using SPSS version 13.0 software program (SPSS Inc., Chicago, Illinois). $P$ values less than 0.05 were considered to be statistically significant.

\section{Results}

3.1. Baseline Clinical Characteristics. As shown in Table 1, T2DM patients had elevated levels of systolic blood pressure (SBP), diastolic blood pressure (DBP), HbAlc, fasting plasma glucose (FPG), and triglycerides (TG) compared to the controls. Subjects with PDR showed markedly decreased HDL-C levels compared with the other three groups.

3.1.1. Serum and Vitreous Omentin-1 Levels. Serum and vitreous omentin-1 levels in the four groups are shown in Table 2. Serum and vitreous omentin-1 levels in PDR patients were both significantly decreased compared with those in the other three groups. There were markedly higher levels of serum and vitreous omentin-1 in the control group compared with the other three groups. Furthermore, NPDR patients showed reduced vitreous omentin-1 levels compared with T2DM patients without DR. However, there were no significant differences in serum omentin-1 levels between NPDR patients and T2DM patients without DR.

3.1.2. Serum/Vitreous Omentin-1 Ratio. Serum/vitreous omentin-1 ratio is presented in Table 2. PDR patients showed significantly elevated serum/vitreous omentin-1 ratio compared with the other three groups. Serum/vitreous omentin-1 ratio was marked increased in NPDR patients compared with the controls and T2DM patients without DR. However, no 
significant differences of serum/vitreous omentin-1 ratio were found between the controls and T2DM patients without DR.

\section{Discussion}

Omentin-1 is a recently discovered adipokine which is preferentially produced by visceral adipose tissue. Omentin-1 treatment could enhance insulin-stimulated glucose uptake in human adipocytes. In addition, omentin increased Akt phosphorylation in human adipocytes [4]. Serum omentin1 levels were lower in the impaired glucose regulation group than in the normal glucose tolerance (NGT) group [6]. Plasma omentin-1 levels were lower in patients with T2DM than in the controls [8]. Furthermore, decreased plasma omentin-1 levels were reported in type 1 diabetes mellitus [9]. Omentin-1 levels were significantly lower in nonobese gestational diabetes mellitus (GDM) women compared to nonobese NGT women [10]. All these findings suggest that omentin-1 may have a protective effect in the pathogenesis of diabetes.

Omentin-1 is associated with the presence of diabetic macrovascular complication. Exposure of cardiomyocytes to conditioned media derived from epicardial adipose tissue from patients with T2DM induced contractile dysfunction and insulin resistance, which was prevented by the addition of recombinant omentin [11]. Yoo et al. reported that T2DM subjects with higher baseline serum omentin-1 levels tended to have a reduced arterial stiffness after one year [12]. In addition, serum omentin-1 levels were significantly decreased in T2DM patients with carotid plaque compared to those without carotid plaque [13].

Omentin-1 is also suggested to be correlated with diabetic microvascular complication. Plasma levels of omentin were found to be markedly higher in end-stage renal disease (ESRD) patients [14]. In another study, omentin-1 levels in patients with nondiabetic chronic kidney disease were found to be higher than the control group [15]. In addition, serum omentin-1 levels in CKD patients were significantly lower compared to the healthy controls. Further analysis revealed that decreased omentin-1 in chronic kidney disease (CKD) patients was due to the reduced omentin-1 levels in the diabetic subgroup [16]. This indicates that serum omentin1 is involved in the mechanism of diabetic nephropathy. DR is another important microvascular complication of diabetes. The present study demonstrated that serum and vitreous omentin-1 levels in PDR patients were both significantly elevated compared with diabetic patients without DR, NPDR patients, and the controls.

Vitreous omentin- 1 concentrations are lower than serum omentin-1 concentrations. This indicates that vitreous omentin-1 may be caused by bleeding from the vascular system of the eyes. This hypothesis could explain the similar reduction of plasma and vitreous omentin-1 concentrations. Omentin1 in eyes may protect the eyes against DR development. And our results showed that serum/vitreous omentin-1 ratio was positively correlated with the presence and severity of DR. This indicates that serum/vitreous omentin-1 ratio may be utilized to predict or assess the development and progression of DR.
Angiogenesis is a key mechanism of DR. Omentin- 1 could significantly decrease vascular endothelial growth factor (VEGF) induced endothelial cell migration and angiogenesis in human microvascular endothelial cells [17]. This suggests that omentin-1 may serve as antiangiogenic mediator and play an important protective role in the development of DR through the antiangiogenic effects.

Omentin-1, one newly discovered adipokine, is shown to be an anti-inflammatory mediator. Omentin was found to inhibit tumor necrosis factor- (TNF-) induced vascular inflammation in human endothelial cells [18] and vascular smooth muscle cells [19]. Serum omentin-1 was reported to be inversely associated with inflammatory cytokines such as TNF- $\alpha$, interleukin-6 (IL-6), and C-reactive protein [20, 21]. These results indicate the anti-inflammatory role of omentin1. Inflammation has been suggested as a potential mechanism for DR. Omentin-1 may be involved in the mechanism of DR via the inhibitory role of inflammatory pathway.

In short, serum and vitreous omentin-1 levels, as well as serum/vitreous omentin-1 ratio, are correlated with the presence and severity of DR.

\section{Conflict of Interests}

The authors declare that there is no conflict of interests regarding the publication of this paper.

\section{References}

[1] World Health Organization, Global Update of Available Data on Visual Impairment, World Health Organization, Geneva, Switzerland, 2006.

[2] J. M. Tarr, K. Kaul, M. Chopra, E. M. Kohner, and R. Chibber, "Pathophysiology of diabetic retinopathy," ISRN Ophthalmology, vol. 2013, Article ID 343560, 13 pages, 2013.

[3] S. Kaštelan, M. Tomić, A. Gverović Antunica, J. Salopek Rabatić, and S. Ljubić, "Inflammation and pharmacological treatment in diabetic retinopathy," Mediators of Inflammation, vol. 2013, Article ID 213130, 8 pages, 2013.

[4] R.-Z. Yang, M.-J. Lee, H. Hu et al., "Identification of omentin as a novel depot-specific adipokine in human adipose tissue: possible role in modulating insulin action," American Journal of Physiology-Endocrinology \& Metabolism, vol. 290, no. 6, pp. E1253-E1261, 2006.

[5] G. Aktas, A. Alcelik, T. Ozlu et al., "Association between omentin levels and insulin resistance in pregnancy," Experimental and Clinical Endocrinology and Diabetes, vol. 122, no. 3, pp. 163-166, 2014.

[6] P. Yan, D. Liu, M. Long, Y. Ren, J. Pang, and R. Li, "Changes of serum omentin levels and relationship between omentin and adiponectin concentrations in type 2 diabetes mellitus," Experimental and Clinical Endocrinology and Diabetes, vol. 119, no. 4, pp. 257-263, 2011.

[7] K. Kazama, T. Usui, M. Okada, Y. Hara, and H. Yamawaki, "Omentin plays an anti-inflammatory role through inhibition of TNF- $\alpha$-induced superoxide production in vascular smooth muscle cells," European Journal of Pharmacology, vol. 686, no. 1-3, pp. 116-123, 2012. 
[8] P. Yan, L. Li, M. Yang et al., "Effects of the long-acting human glucagon-like peptide-1 analog liraglutide on plasma omentin1 levels in patients with type 2 diabetes mellitus," Diabetes Research and Clinical Practice, vol. 92, no. 3, pp. 368-374, 2011.

[9] B. K. Tan, S. Pua, F. Syed, K. C. Lewandowski, J. P. O’Hare, and H. S. Randeva, "Decreased plasma omentin-1 levels in Type 1 diabetes mellitus," Diabetic Medicine, vol. 25, no. 10, pp. 12541255, 2008.

[10] G. Barker, R. Lim, H. M. Georgiou, and M. Lappas, "Omentin1 is decreased in maternal plasma, placenta and adipose tissue of women with pre-existing obesity," PLoS ONE, vol. 7, no. 8, Article ID e42943, 2012.

[11] S. Greulich, W. J. Y. Chen, B. Maxhera et al., "Cardioprotective properties of omentin-1 in type 2 diabetes: evidence from clinical and in vitro studies," PLoS ONE, vol. 8, no. 3, Article ID e59697, 2013.

[12] H. J. Yoo, S. Y. Hwang, H. C. Hong et al., "Implication of circulating omentin-1 level on the arterial stiffening in type 2 diabetes mellitus," Endocrine, vol. 44, no. 3, pp. 680-687, 2013.

[13] H. J. Yoo, S. Y. Hwang, H. C. Hong et al., "Association of circulating omentin-1 level with arterial stiffness and carotid plaque in type 2 diabetes," Cardiovascular Diabetology, vol. 10, article 103, 2011.

[14] A. Alcelik, M. Tosun, M. F. Ozlu et al., "Serum levels of omentin in end-stage renal disease patients," Kidney \& Blood Pressure Research, vol. 35, no. 6, pp. 511-516, 2012.

[15] E. Sengul, G. Duygulu, S. Dindar, and F. Bunul, "Serum omentin-1, inflammation and carotid atherosclerosis in patients with non-diabetic chronic kidney disease," Renal Failure, vol. 35, no. 8, pp. 1089-1093, 2013.

[16] H. Tekce, B. K. Tekce, G. Aktas, A. Alcelik, and E. Sengul, "Serum omentin-1 levels in diabetic and nondiabetic patients with chronic kidney disease," Experimental and Clinical Endocrinology \& Diabetes, vol. 122, pp. 451-456, 2014.

[17] B. K. Tan, R. Adya, S. Farhatullah, J. Chen, H. Lehnert, and H. S. Randeva, "Metformin treatment may increase omentin-1 levels in women with polycystic ovary syndrome," Diabetes, vol. 59, no. 12, pp. 3023-3031, 2011.

[18] H. Yamawaki, J. Kuramoto, S. Kameshima, T. Usui, M. Okada, and Y. Hara, "Omentin, a novel adipocytokine inhibits TNFinduced vascular inflammation in human endothelial cells," Biochemical and Biophysical Research Communications, vol. 408, no. 2, pp. 339-343, 2011.

[19] K. Kazama, T. Usui, M. Okada, Y. Hara, and H. Yamawaki, "Omentin plays an anti-inflammatory role through inhibition of TNF- $\alpha$-induced superoxide production in vascular smooth muscle cells," European Journal of Pharmacology, vol. 686, no. 1-3, pp. 116-123, 2012.

[20] H.-Y. Pan, L. Guo, and Q. Li, "Changes of serum omentin-1 levels in normal subjects and in patients with impaired glucose regulation and with newly diagnosed and untreated type 2 diabetes," Diabetes Research and Clinical Practice, vol. 88, no. 1, pp. 29-33, 2010.

[21] J. M. Moreno-Navarrete, F. Ortega, A. Castro, M. Sabater, W. Ricart, and J. M. Fernández-Real, "Circulating omentin as a novel biomarker of endothelial dysfunction," Obesity, vol. 19, no. 8, pp. 1552-1559, 2011. 


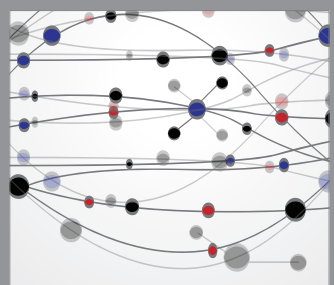

The Scientific World Journal
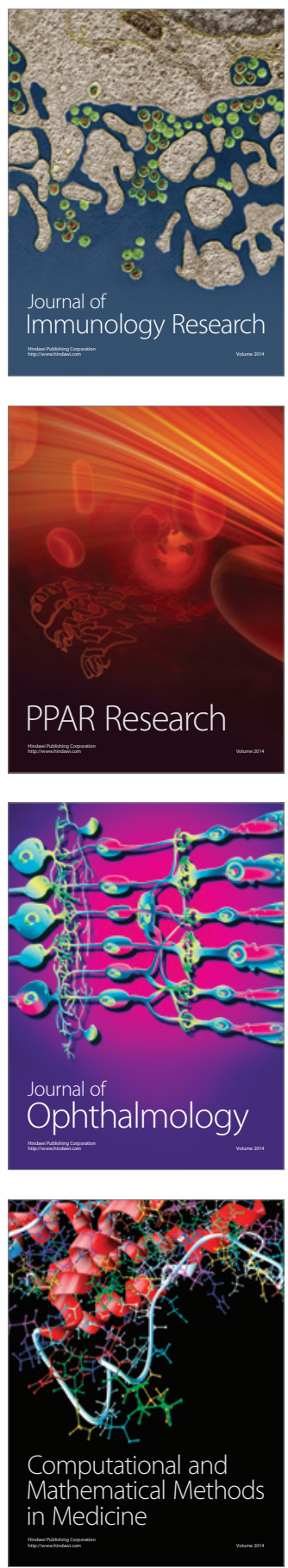

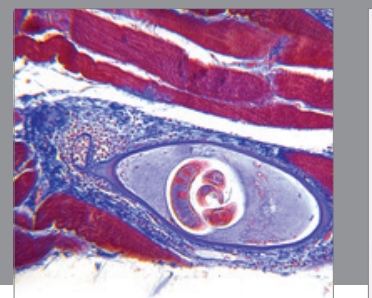

Gastroenterology

Research and Practice
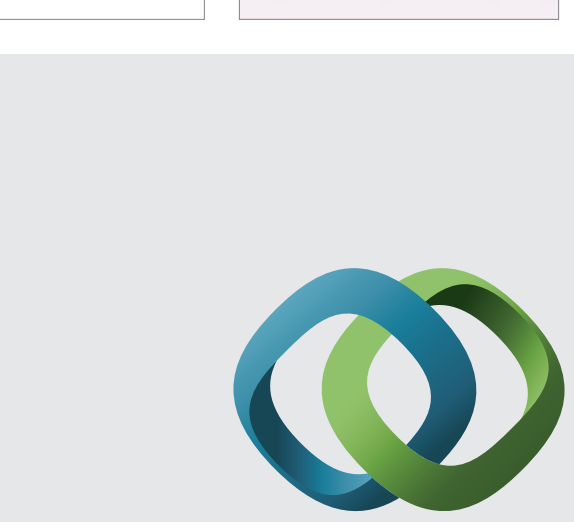

\section{Hindawi}

Submit your manuscripts at

http://www.hindawi.com
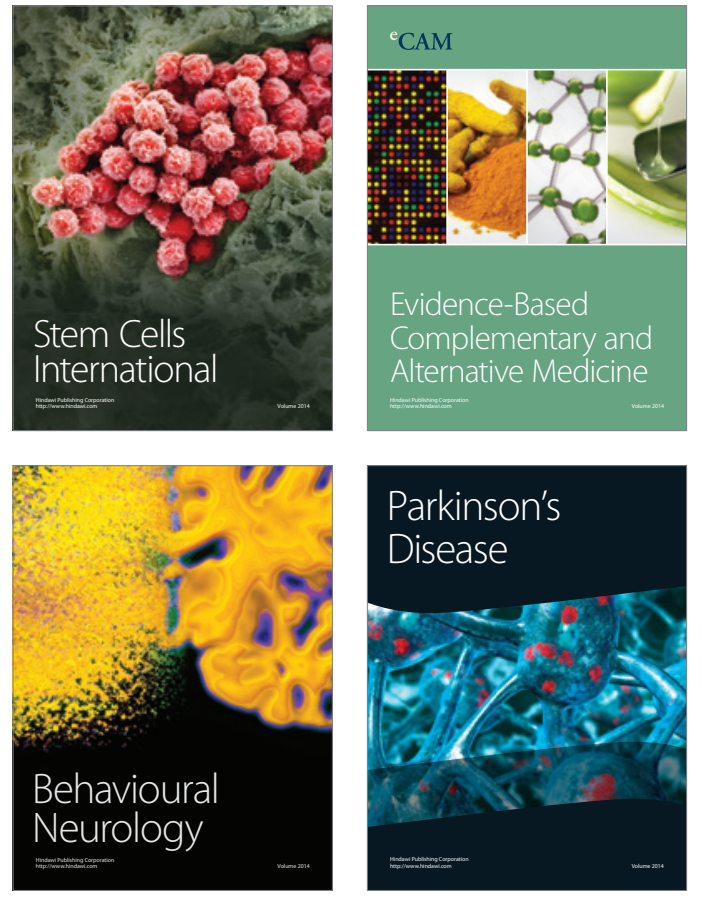
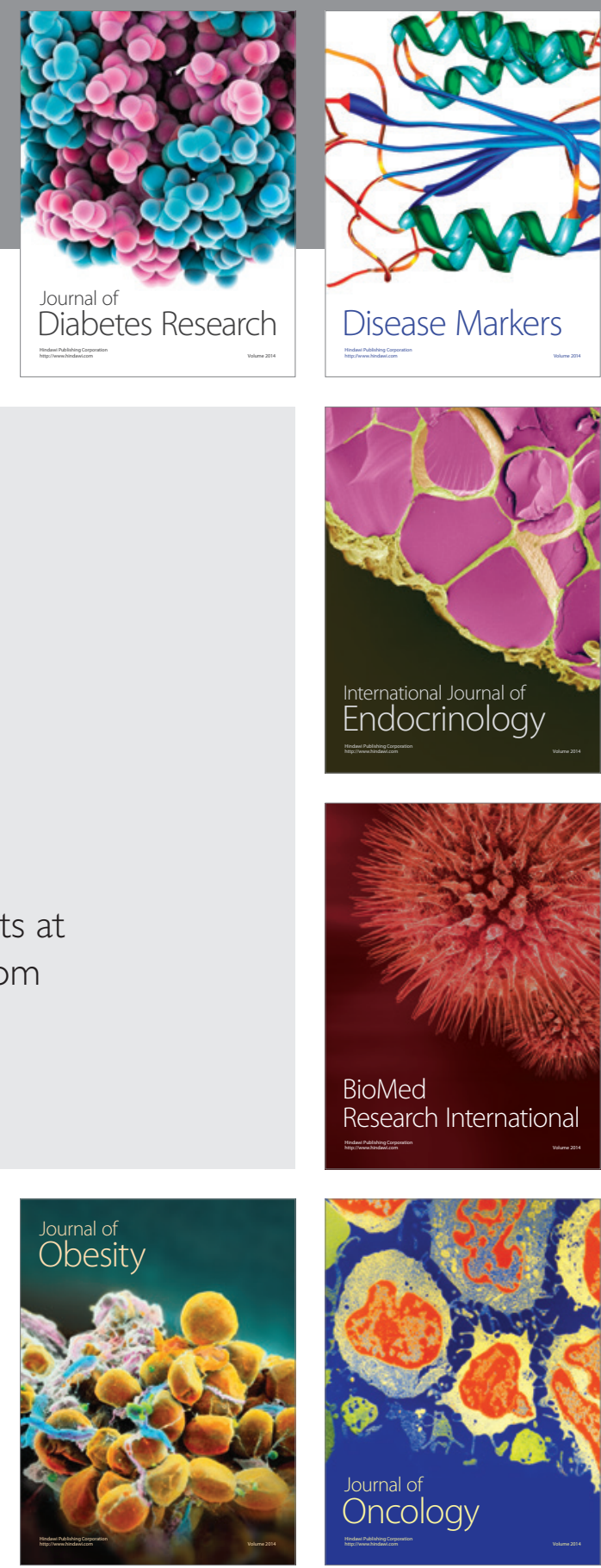

Disease Markers
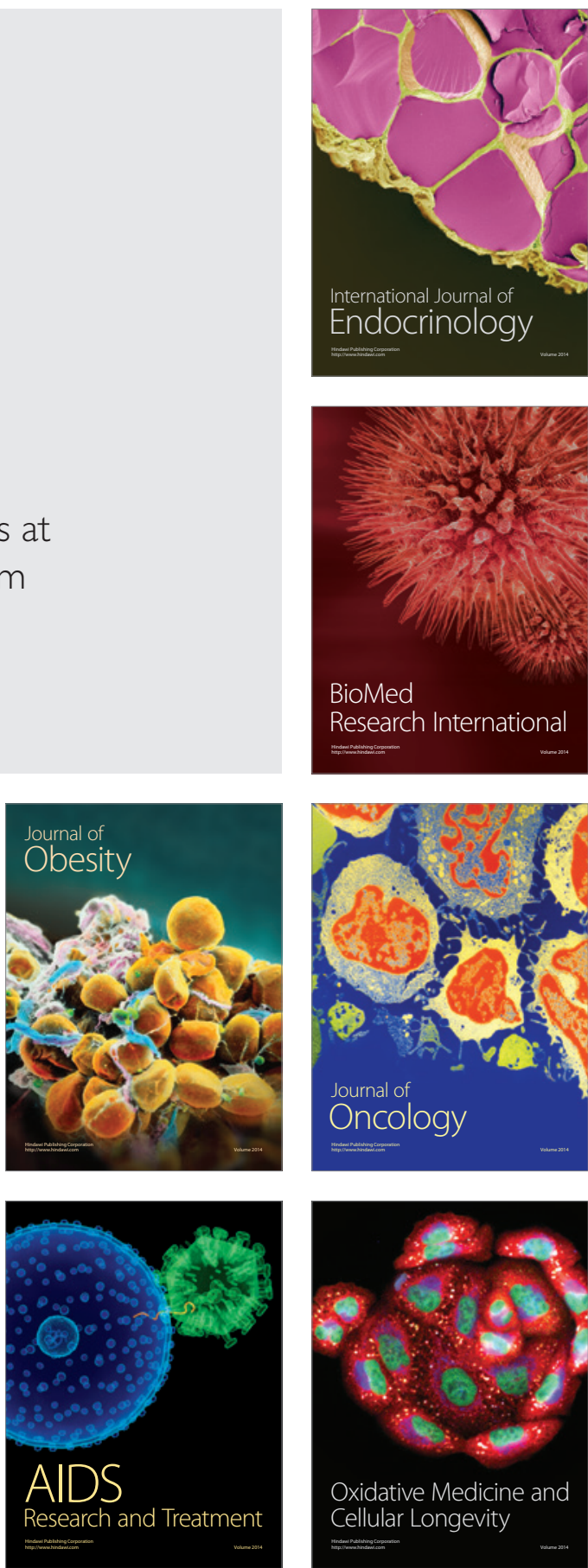